\title{
Expression of eNOS in kidneys from hypertensive patients
}

This article was published in the following Dove Press journal: International Journal of Nephrology and Renovascular Disease 6 March 2010

Number of times this article has been viewed

\section{Xin Gu \\ Guillermo A Herrera \\ 'Department of Pathology, Louisiana State University Health Sciences Center, Shreveport, LA, USA; ${ }^{2}$ Nephrocor, Bostwick Laboratories, Tempe, AZ, USA}

\begin{abstract}
Endothelium-derived nitric oxide (NO) is essential for maintenance and regulation of blood pressure. In animal models, altered endothelium-derived nitric oxide synthase (eNOS) expression and impaired NO generation are important factors for renal injury. However, the pattern of eNOS expression in the kidneys from hypertensive patients has not been well established. We have studied the eNOS immuno-expression in kidney biopsies from hypertensive patients. Compared to kidneys from normotensive individuals, there were no significant alterations of eNOS immuno-expression in the vasculature of patients with chronic essential hypertension. In contrast, the expression of eNOS was significantly decreased in the glomeruli and arterioles/small arteries of patients with malignant hypertension, particularly in those with significant intimal edema and myxoid degeneration or thrombi. Endothelial dysfunction is an important pathogenetic factor for chronic primary hypertension and eNOS plays a major role in the regulation of vascular tone and function. Unchanged eNOS in the kidney vasculature in chronic primary hypertension indicates that these patients have an ability to compensate. In patients with malignant hypertension, the expression of eNOS protein was diminished in the injured vasculature. Loss of the compensatory mechanism via continued release of NO to prevent vascular injury may be responsible for morphological changes typically seen in the renal vasculature in patients with accelerated hypertension.
\end{abstract}

Keywords: endothelium-derived nitric oxide, kidney, chronic hypertension, animal models

\section{Introduction}

Primary or essential hypertension is characterized by chronically elevated blood pressure of unknown etiology. This is one of the most important risk factors for developing chronic kidney disease. Normal renal blood flow in concert with the renal angiotensin system plays a critical role in the maintenance of physiological hemostasis and adjustment of blood pressure. The importance of the endothelial isoform of nitric oxide synthase (eNOS) in maintaining normal renal hemodynamics has been well established. ${ }^{1-4}$ Endothelial function is mainly dependent on the generation and bioavailability of NO produces by eNOS. In animal studies, reduced NO level is a common denominator of many hypertensive models $\mathrm{s}^{5,6}$ and direct inhibition of intrarenal NO produce an increase in blood pressure. ${ }^{7}$ Impaired endothelial NO generation is considered one of the important factors associated with hypertension and atherosclerosis that result in progressive renal injury. ${ }^{8,9}$ In addition, expression of eNOS and bioavailability of NO may be altered by a variety of signal transduction molecules, receptors for growth factors, and cytokines. ${ }^{10,11}$ In animal models, enhanced expression of eNOS has been shown to be associated with resistance to hypertensive stimulation. ${ }^{12-14}$
Correspondence: Xin Gu

Department of Pathology, Louisiana State University Health Sciences Center,

I50I Kings Highway, Shreveport,

LA 7II30, USA

Emailxgu@Isuhsc.edu submit your manuscript | www.dovepress.com

Dovepress 
As an important modulator of renal function and morphology, the NO system has been extensively studied in the animal models and clinical settings. However, despite increasing evidence for the role of eNOS and NO, little is known about eNOS expression in the human kidneys in patients who had long-standing hypertension. The main objective of this study is to use immunohistochemical methods to investigate the expression of eNOS in the kidneys from patients with chronic primary hypertension, and alterations of eNOS expression in patients with chronic essential hypertension who developed accelerated or malignant hypertension.

\section{Materials and methods}

\section{Selection of cases and tissue processing}

Normal kidney controls were collected from five adult normotensive patients who had nephrectomy for excision of renal neoplasms. The control specimens were collected from renal parenchyma that was uninvolved by neoplasms (renal cell carcinomas). Six kidney biopsies from adult patients with chronic primary hypertension and five kidney biopsies from patients with malignant hypertension were retrospectively identified from our archives of kidney biopsies.

All kidney biopsies were processed for light microscopy, direct immunofluorescence, and electron microscopy according to standard techniques. ${ }^{15,16}$ The controls were processed for light microscopy only. No immunofluorescence or electron microscopic evaluation was performed in these samples.

\section{Immunohistochemistry}

Immunohistochemical stains were performed on formalin-fixed, paraffin-embedded tissue sections. A standard immunohistochemistry staining procedure was performed as we previously described. ${ }^{17}$ Briefly, deparaffinization was performed using xylene and ethanol. Antigen retrieval was achieved by boiling tissue slides with $0.01 \mathrm{M}$ citric buffer in a microwave for five minutes. Hydrogen peroxide was used to quench the endogenous peroxidase activity.

After blocking with 10\% serum-tris buffer for 20 minutes at room temperature, the sections were incubated with primary polyclonal anti-eNOS antibody (1:50 dilution in Tris buffer; Abcam, Cambridge, MA) at room temperature for 120 minutes. Corresponding biotinylated conjugated secondary antibody from Dako staining system was used. Slides stained with secondary antibody only were used as negative controls. The nuclei were counterstained with hematoxylin.

The intensity of immunostaining for eNOS in endothelial cells was evaluated on a 0 to $3+$ scale $(0$, no staining; $1+$, weak staining; 2+, moderate - easily identified in the cytoplasm using high magnification $-60 \times$; and $3+$, strong staining that was easily identified from low power magnification $-20 \times$ ).

\section{Results \\ Clinical data}

All kidney specimens were from adult patients (aged 24 to 76 years). In the control group, the patients had no known clinical history of hypertension. Their pre-operative blood pressures were within normal limits.

The clinical data from the control group and from, patients with chronic primary and malignant hypertension are summarized in Table 1. All patients in the chronic hypertension group had a history of primary hypertension for more than five years. The biopsies were performed for deterioration of renal function with or without proteinuria. All patients with malignant hypertension had a history of hypertension for at least two years. Three of these patients presented with sudden onset of headache, nausea, elevated blood pressure over $210 / 120$ and, variable degrees of renal insufficiency. One presented to the emergency room with an infectious process and was incidentally identified with accelerated hypertension (blood pressure $=190 / 110$ ) and an elevated serum creatinine. Another patient had blurry vision and was found to have markedly elevated blood pressure (over 210/120), as well as an increased serum creatinine. In both primary hypertension and malignant hypertension groups, laboratory work up revealed negative serology tests, including anti-nuclear antibodies (ANA) and antineutrophilic cytoplasmic antibodies (ANCA). They had all been in treatment with a variety of antihypertension medications for variable periods of time.

\section{Pathology findings}

Control kidneys were normal. There was no significant interstitial fibrosis, tubular atrophy or thickening of vascular intima. The glomeruli showed open loops. The capillary walls were thin and smooth (Figure 1A). There was no fibrinoid thickening of the intima of arteriole and interlobular arteries (Figure 2A). The kidneys from patients in the chronic primary hypertensive group showed patchily distributed interstitial fibrosis associated with tubular atrophy and drop out. Scattered lymphocytes were often present in association with areas with interstitial fibrosis. The viable glomeruli showed retraction of capillary tufts with segmental thickening and wrinkling of capillary loops (Figure 1B). The interlobular arteries revealed intimal fibrous thickening and the arterioles showed thickening of their walls with segmental hyalinosis (Figure 2B). Except for the vascular disease-associated 
Table I Demographic and clinical data

\begin{tabular}{|c|c|c|c|c|c|}
\hline \multicolumn{2}{|l|}{ Control (5 cases) } & \multicolumn{2}{|c|}{ Chronic hypertension (6 cases) } & \multicolumn{2}{|c|}{ Malignant hypertension (5 cases) } \\
\hline & $57.6 \pm 3.6$ & Age, mean $\pm S D$ & $58.7 \pm 11.9$ & Age, mean $\pm S D$ & $41.6 \pm 11.9$ \\
\hline Age, range & 53 to 63 & Age, range & 41 to 76 & Age, range & 24 to 55 \\
\hline Sex, male/female & $3 / 2$ & Sex, male/female & $4 / 2$ & Sex, male/female & $4 / 1$ \\
\hline Proteinuria & & Proteinuria & & Proteinuria & \\
\hline Trace & 0 & Trace & 2 & Trace & I \\
\hline Nonnephrotic & 0 & Nonnephrotic & 2 & Nonnephrotic & 2 \\
\hline Nephrotic & 0 & Nephrotic & 0 & Nephrotic & 0 \\
\hline Data not available & 0 & Data not available & 2 & Data not available & 2 \\
\hline Serum creatinine (mg/dL) & & Serum creatinine $(\mathrm{mg} / \mathrm{dL})$ & & Serum creatinine $(\mathrm{mg} / \mathrm{dL})$ & \\
\hline Normal & 5 & Normal & 2 & Normal & 0 \\
\hline Abnormal & 0 & Abnormal & 2 (less than 2 ) & Abnormal & 4 (3.7 to I2) \\
\hline Data not available & 0 & Data not available & 2 & Data not available & I \\
\hline Microhematuria & 0 & Microhematuria & 0 & Microhematuria & 2 \\
\hline Data not available & & Data not available & & Data not available & 3 \\
\hline History of CRI* & 0 & History of CRI & 0 & History of CRI & 2 \\
\hline Data not available & & Data not available & & Data not available & 3 \\
\hline Blood pressure & & Blood pressure & & Blood pressure & \\
\hline Above $140 / 90$ & 0 & Above $140 / 90$ & 6 & Systolic & 190 to 220 \\
\hline & & & & Diastolic & 110 to 140 \\
\hline
\end{tabular}

Abbreviation: $\mathrm{CRI}$, chronic renal insufficiency.

changes, there was no morphologic evidence of a specific glomerular disorder, such as proliferative glomerulonephritis, immune complex mediated glomerulonephritis or diabetic glomerulopathy. There was no evidence of tubular necrosis or interstitial nephritis. Ultrastructurally, segmental wrinkling and thickening of capillary basement membranes were common in glomeruli. There were no significant endothelial structural alterations. No immune complexes or paraprotein deposits were identified in any of the renal compartments in any of these cases.

In patients with malignant hypertension, the interstitium revealed similar findings as in the chronic hypertensive patients, but more pronounced. In the glomeruli, the morphologic findings were variable. Some glomeruli revealed typical features of thrombotic microangiopathy with segmental fibrinoid necrosis of capillary tufts (Figure 1C). Some glomeruli showed widening of subendothelial spaces and mesangiolysis, while other glomeruli were essentially unremarkable under light microscopic evaluation. The pathologic findings in the glomeruli were often highlighted by periodic acid-Schiff(PAS) and silver methenamine stains that stained basement membranes. ${ }^{15,16}$ The arterioles and small arteries revealed significant intimal thickening and myxoid change (Figure 2C). Hyperplastic arteriolopathy ("onion-skinning") was present in all cases, and arteriolar fibrinoid necrosis was identified in two cases (Figure 1C). By electron microscopy, there was widening of subendothelial spaces with electron lucent fluffy-appearing material in the glomeruli. Mesangiolysis, fragmented platelets and red blood cells, and fibrin were identified in the subendothelium in some glomeruli (Figure 1D).

\section{Expression of eNOS}

In the control group (Figure 3A), moderate to strong (3+) expression of eNOS was present in the cytoplasm of endothelial cells in glomeruli, arterioles, interlobular arteries, veins and peritubular capillaries (Figure 4A). In the glomeruli, there was no immunoreaction in mesangial cells. The visceral epithelial cells that were also negative for eNOS. Weak staining along tubular basement membranes and cytoplasm of some tubular epithelial cells ( 0 to $1+$ ) was noted in focal areas. The interstitial cells and inflammatory cells were negative for eNOS.

The expression of eNOS in biopsies from primary hypertensive patients revealed similar findings as that in the controls. There was no significant difference in the patterns of expression and the intensity of immunoreaction in glomeruli (Figure 3B), arterioles, and small arteries (Figure 4B). In the interstitium, when there were dense lymphocytic infiltrates, the peritubular capillaries in the nearby areas 

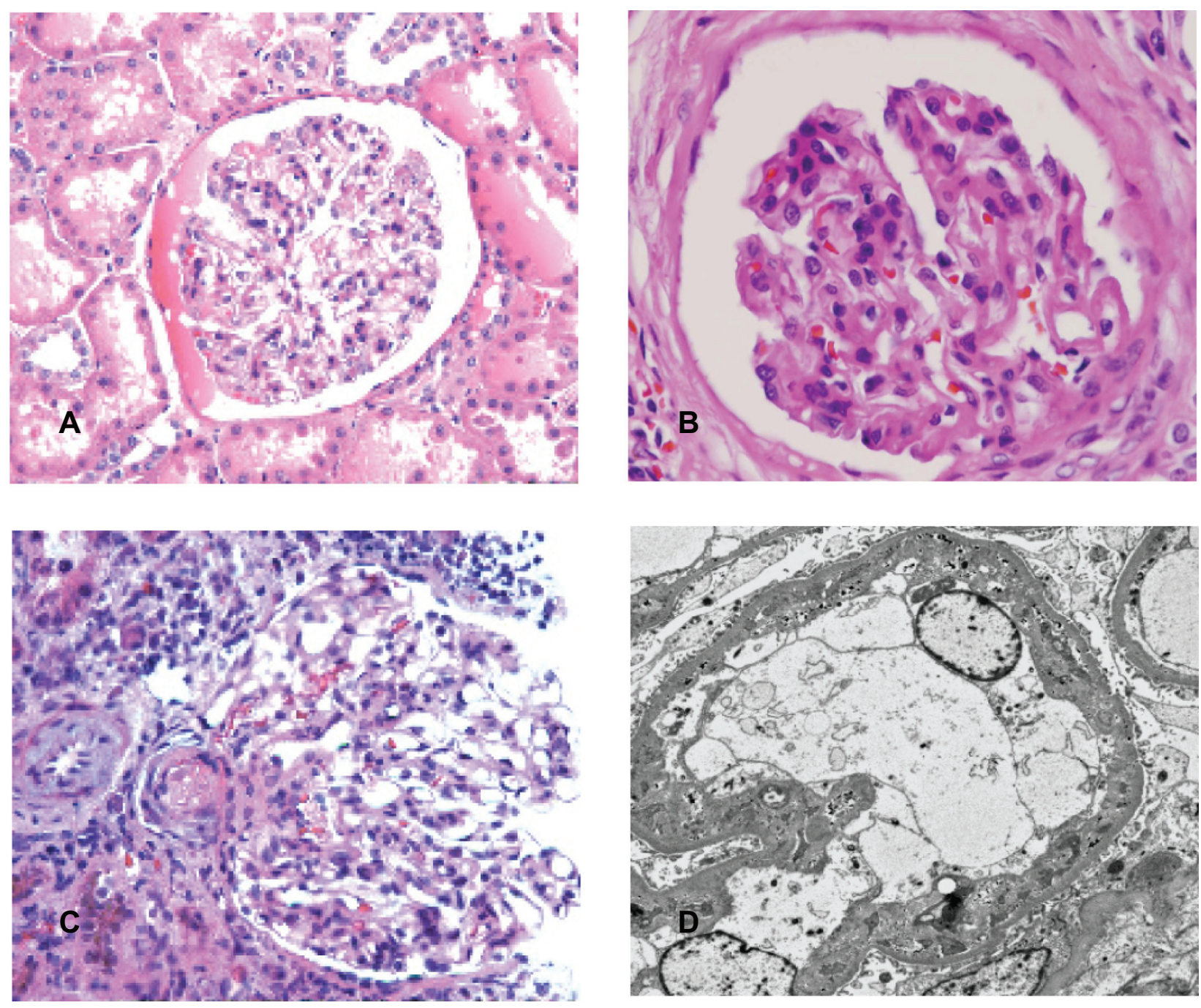

Figure I Glomeruli. A) Glomerulus from control kidney. B) Glomerulus from patient with chronic hypertension. The glomerulus revealed retraction of capillary tufts and segmental wrinkling of membranes. C) Glomerulus from patient with malignant hypertension. The glomerulus showed segmental fibrinoid necrosis of capillary tufts. Microthrombus was present in the arteriole. D) Electron microscopy of glomerulus from patient with malignant hypertension. There was widening of subendothelial spaces with accumulation of fragmented platelets and fibrin.

expressed relatively strong immunoreactivity for eNOS when compared to the capillaries that were not associated with the surrounding interstitial inflammation.

In patients with malignant hypertension, the expression of eNOS was significantly attenuated in the glomeruli (Figure 3C), arterioles and small arteries. In the glomeruli and arterioles that showed prominent intima edema or fibrinoid thrombi, eNOS expression was diminished (Figure 4C). The patterns and intensity of eNOS in the tubulointersitium appeared similar to that in the chronic hypertensive patients.

\section{Discussion}

Primary hypertension is a common disease. Uncontrolled hypertension is one of the most important risk factors for cardiovascular disease, stroke and sudden death. Chronic hypertension is also a leading cause for developing end-stage renal disease. Malignant hypertension may suddenly appear in patients with chronic hypertension and is life threatening. Renal endothelial cell integrity and vascular homeostasis play a critical role in monitoring and maintaining normal vascular tone and blood pressure. ${ }^{1,9}$

In general, vascular endothelial cells release NO under normal (basal) conditions as a result of shear stress exerted by the circulating blood. The released NO diffuses to the underlying vascular smooth muscle cells and activates soluble guanylate cyclase, leading to an increase in cGMP resulting in relaxation. ${ }^{18,19}$ Together with vasoconstrictive mediators, renal endothelial cell-derived NO plays a critical role in regulation of renal blood flow and 

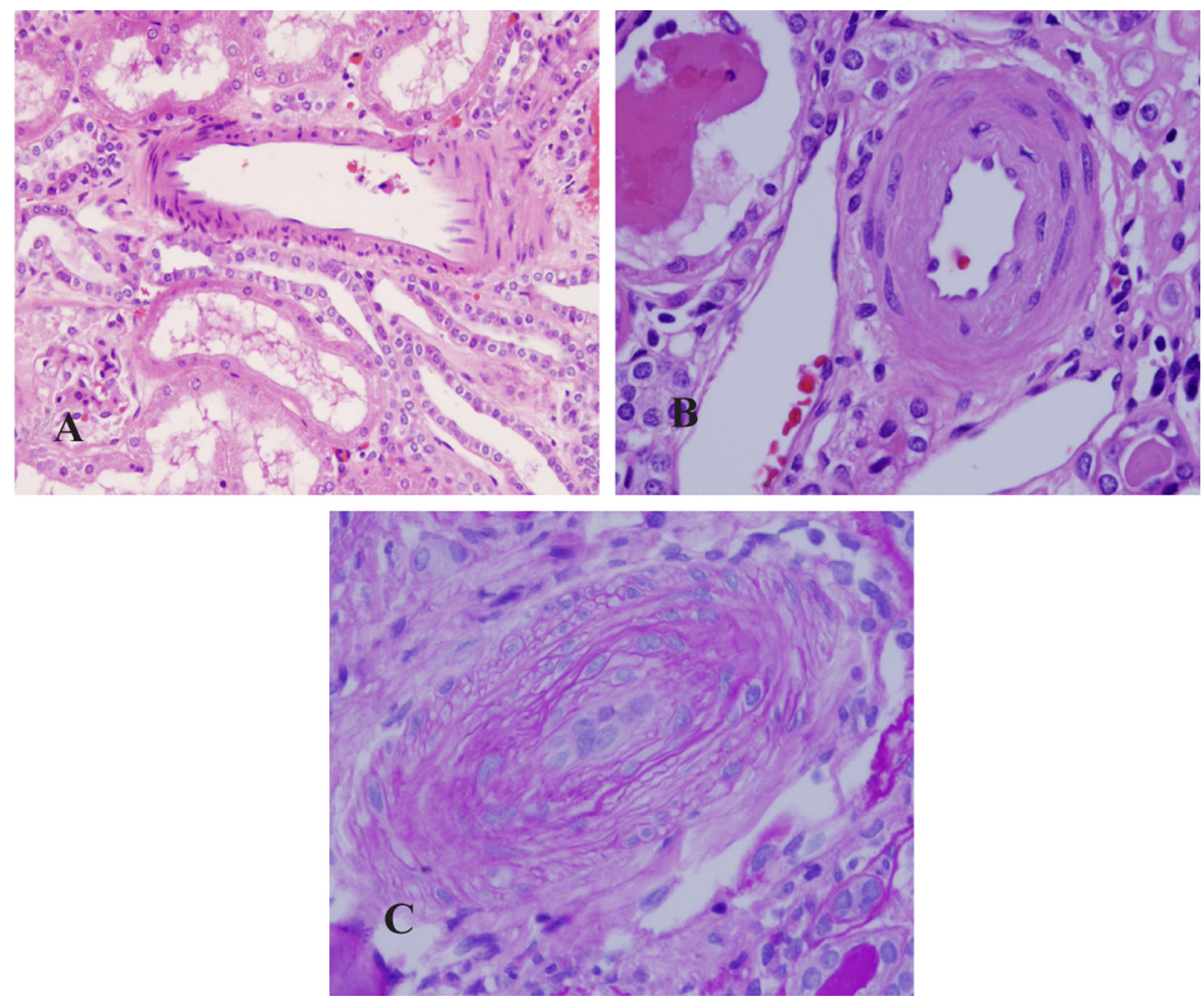

Figure 2 Small arteries. A) A small artery in control kidney. B) A small artery in the kidney from patient with chronic hypertension.The artery showed thickening and hyalinosis of vascular wall. C) A small artery in the kidney from patient with malignant hypertension. The artery revealed significant thickening of vascular wall, myxoid degeneration of intima and obliteration of lumen. The endothelial cells showed edema with enlarged nuclei and pale cytoplasm.

glomerular filtration rate. Endothelial NOS produced NO is also responsible for modulation of angiotensin II-mediated renal norepinephrine release. ${ }^{4,20}$ In addition, NO released from endothelial cell also has antiplatelet, antiproliferation, and anti-inflammatory properties. ${ }^{21,22}$

It has been well documented that renal endothelial dysfunction, characterized by decreased production or bioavailability of $\mathrm{NO}$, is associated with the pathogenesis of hypertension. In animal models, lower levels of eNOS activity resulted in vasoconstriction with an increase in blood pressure. ${ }^{23,24}$ Low NO levels were also associated with vascular hypertrophy, intimal thickening, and reduced vascular lumina. ${ }^{25}$ Studies performed in humans have indicated that hypertension is associated with selective impairment of endothelium-dependent vasodilation. ${ }^{9}$
Expression of eNOS and production of $\mathrm{NO}$ are regulated at multiple levels and through different pathways. Shear stress is the major factor for inducing eNOS upregulation. In contrast, hypoxia, tumor necrosis factor- $\alpha$, and oxygen radicals downregulate eNOS expression. ${ }^{1,11}$ Second messenger systems, intracellular calcium and phosphorylation are involved in regulation of eNOS. ${ }^{1,26}$ In addition, NO bioavailability is also adjusted by the rate of scavenger activity, such as regional radical oxygen species. ${ }^{8}$

Although plenty of data have been generated supporting that deficiency of eNOS and bioavailability of NO are related to the development of hypertension and persistence of elevated blood pressure, in most instances the information was obtained from animal models or in vitro studies. There is little information on eNOS expression in the kidneys in 

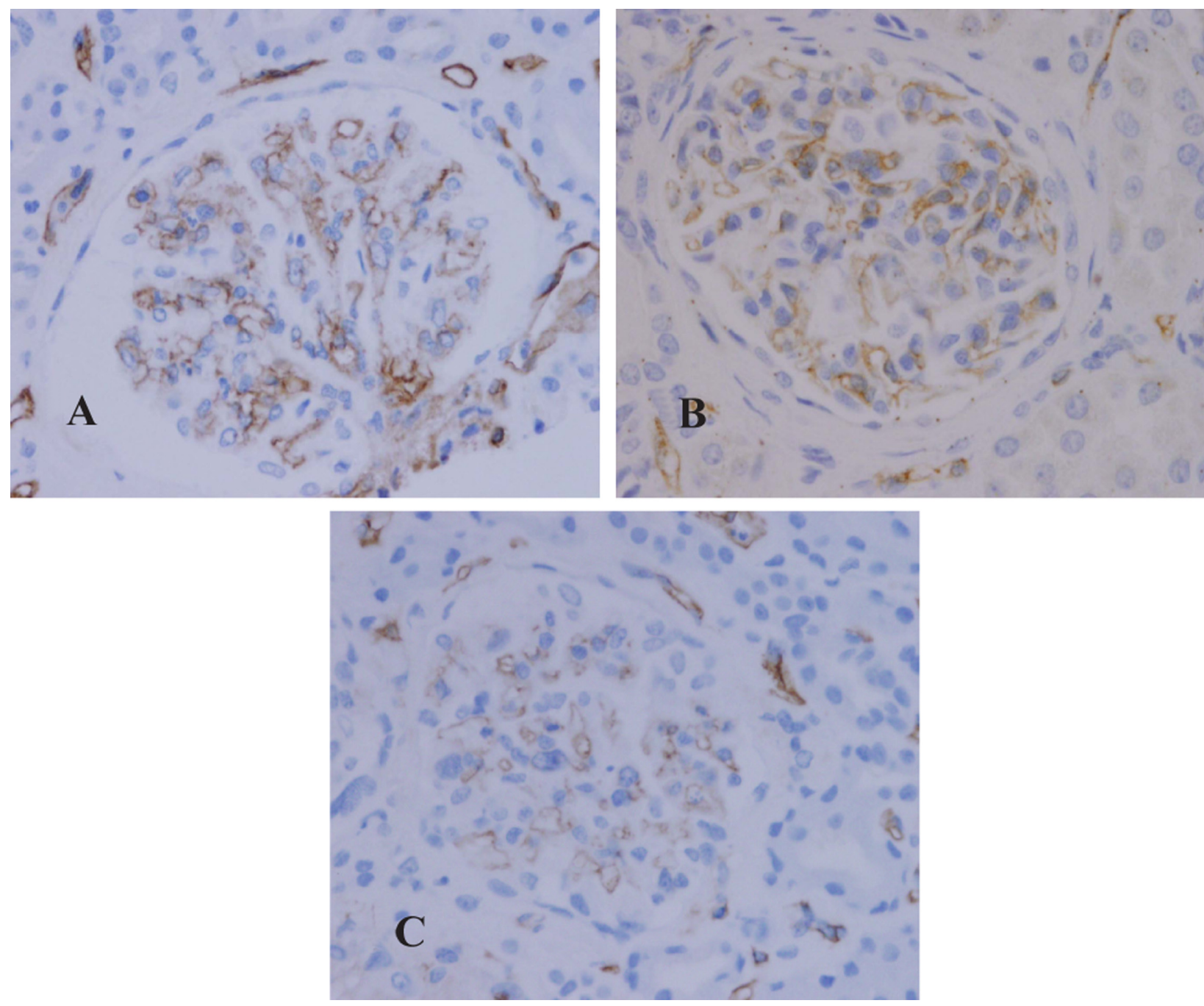

Figure 3 Expression of eNOS in the glomeruli. A) Control. B) Chronic hypertension. C) Malignant hypertension.

patients with long-term chronic hypertension. In this study, we have detected eNOS protein expression in the kidneys in patients with chronic primary hypertension, as well as in chronic hypertensive patients who had developed accelerated or malignant hypertension.

In this immunohistochemical study, moderate to strong (2-3+) eNOS expression was identified in the endothelial cells in all locations, including glomeruli, arterioles, small arteries, interlobular arteries and veins as well as peritubular capillaries (Figures 3A, 4A). There was no significant difference in terms of intensity of immuno-expression in the different-sized vessels. Compared to the normal control group, there was no alteration of eNOS expression in the glomeruli (Figure 3B) and vessels from patients with chronic primary hypertension (Figure 4B). In both groups, there was moderate to strong eNOS immunoreaction in the endothelial cells. However, the expression of eNOS was attenuated in the endothelial cells in the glomeruli and arterioles from patients with malignant hypertension. Only minimal to mild immunoreactivity (1+) was noted in the glomeruli (Figure 3C) and arterioles in the kidneys from patients in malignant hypertension. Moderate immunoreactivity for eNOS was only occasionally identified in glomerular epithelial cells. In the vasculature exhibiting microthrombi and fibrinoid necrosis, the eNOS immunoreactivity was markedly diminished (Figure 4C).

Glomerular endothelial cells are often injured in patients with malignant hypertension. Histologically, glomerular segmental fibrinoid necrosis with or without microthrombi may be seen associated with the characteristic hyperplastic arteriolopathy. The glomeruli without significant light microscopic changes may also reveal endothelial cell injury that can be only detected at the ultrastructural level, including edema of endothelial cells, fragmentation of the cytoplasm 

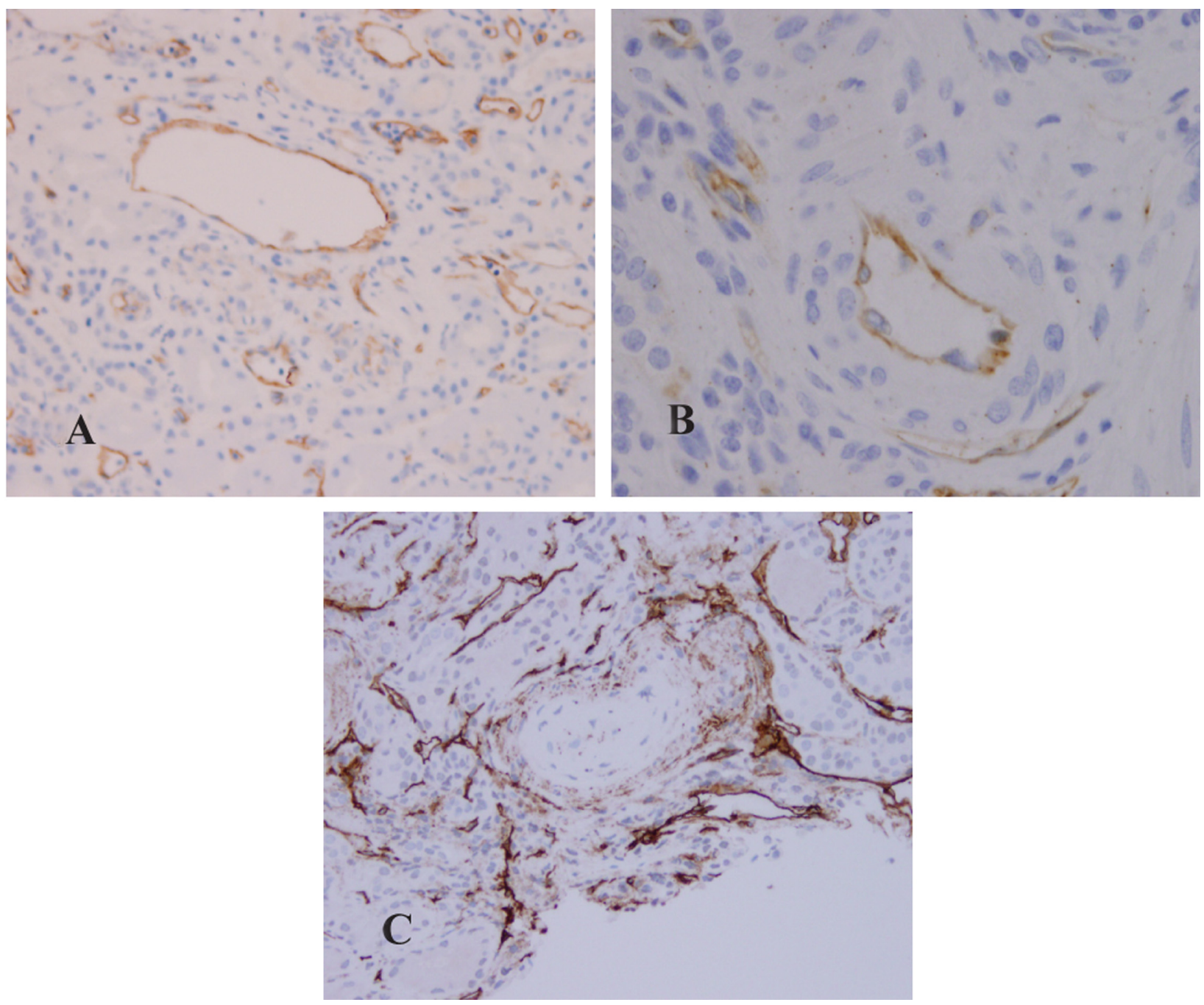

Figure 4 Expression of eNOS in the arteries. A) Control. B) Chronic hypertension. myxoid degeneration of intima and injured endothelial cells.

and accumulation of electron lucent material in the subendothelial spaces. In addition, immunoreactivity for receptors and important enzymes may also be altered, as we recently reported in kidney biopsies from pregnant women with significantly elevated blood pressures. ${ }^{17}$

These results suggest that, compared to the control group, there is no alteration of eNOS protein expression in chronic primary (essential) hypertension. However, expression of eNOS is attenuated in association with accelerated (malignant) hypertension. The attenuated expression of eNOS is most likely related to more severe endothelial injury in malignant hypertension cases.

In this study, the control specimens were collected from nephrectomy kidney from patients with neoplasms. The specimens were taken at some distance from the neoplasms as previous studies reported. ${ }^{27,28}$ Except for uncommon neoplasms (renal juxtaglomerular cell tumor and carcinoid tumor), renal neoplasms do not affect systemic circulation and blood pressure. As seen in Table 1, the age range in hypertensive patients was broad. This may reflect the fact that hypertension is a common disease and affects a wide range of the population. In both primary and malignant hypertension groups, patients had renal function dysfunction and presented with variable degrees of proteinuria and/or elevated serum creatinine. The pathological findings from these patients are more likely due to persistent hypertension rather than merely aging.

Protein expression does not necessarily correlate with enzyme activity and NO production. In chronic primary hypertensive patients, dysfunction of renal endothelial cells, alteration in NO synthesis, and release may occur mainly post-transcriptionally, resulting from such processes as abnormal phosphorylation, uncoupling with second messenger systems, and NO scavenging, as has been 
suggested in previous studies performed in animal models and hypertensive patients. ${ }^{29,30}$ Genetic polymorphism of eNOS may also contribute to reduced bioavailability of NO, as recent studies have suggested. ${ }^{31,32}$ In the biopsies from malignant hypertension patients, the expression of eNOS was decreased in the injured vasculature.

Based on this study, we assume that in chronic essential hypertension it is likely that eNOS-derived NO remains at a critical (minimal) level which maintains hemodynamic balance. In this condition the endothelial cells are dysfunctional and their compensatory ability is limited. Factors that trigger unbalanced NO bioavailability induce rapid blood pressure elevation, as has been demonstrated in studies performed on spontaneously hypertensive rats. ${ }^{33}$ The decrease of bioavailable NO further precipitates endothelial injury, platelet aggregation with formation of microthrombi, and endothelial cell necrosis, as has been reported to occur in some glomerulonephritis in human studies and animal models. ${ }^{28,34,35}$ The totally absent eNOS immunoreactivity in the severe form of thrombotic angiopathy was most likely a consequence of endothelial cell necrosis.

Previous studies have also indicated that inducible NOS (iNOS) is present in the kidneys. The expression of iNOS has been documented in tubular epithelial and inflammatory cells. Inducible-NOS has also been shown in mesangial cells after exposure to cytokines or endotoxins, but not in the normal renal tissue. ${ }^{35,36}$ The expression of iNOS has been demonstrated to be significantly increased in the glomeruli in lupus nephritis and in experimental antimyeloperoxidase-associated crescentic glomerulitis. ${ }^{35,37,38}$ In transplant kidneys, iNOS has been shown to participate in ischemia-reperfusion-induced tubular and glomerular injury. ${ }^{39,40}$ In these conditions, excessive amount of NO was generated by iNOS and increased NO radical production resulted in tissue injury, as revealed in experimental animal models. In general iNOS is not directly involved in the pathophysiology of hypertension or in adjusting vascular tone in the kidney.

Clinically, hypertension is treated with a variety of medications, such as diuretics, calcium-channel blockers and angiotensin-converting enzyme inhibitors. Some of these medications may affect NO bioavailability, ${ }^{41}$ however, there is no evidence that these medications affect eNOS expression. In recent studies, effective delivery of NOS gene to endothelial cells has demonstrated therapeutic benefits in the animal models. ${ }^{42,43}$ These studies suggest that enhancement of eNOS expression though gene therapy may be a useful therapeutic modality for hypertension.
In conclusion, eNOS plays a major role in the regulation of vascular tone and function, and endothelial dysfunction is an important pathogenetic factor for chronic primary hypertension. Endothelial NOS was unchanged in the kidney vasculature at the protein level in patients with chronic primary hypertension. In patients with malignant hypertension, the expression of eNOS protein was decreased or diminished in the injured glomeruli, small renal arteries and arterioles. In these patients, enhanced vasoconstrictive response and loss of compensatory mechanisms (via continued NO release from endothelial cells to prevent vascular injury and rapid acceleration) may be responsible for progression into malignant hypertension.

\section{Disclosures}

The authors report no conflicts of interest in this work.

\section{References}

1. Mount PF, Power DA. Nitric oxide in the kidney: functions and regulation of synthesis. Acta Physiol. 2006;187:433-446.

2. Kone BC. Nitric oxide synthesis in the kidney: isoforms, biosynthesis, and functions in health. Semin Nephrol. 2004;24:299-315.

3. Desjardins F, Balligand JL. Nitric oxide-dependent endothelial and cardiovascular disease. Acta Clin Belg. 2006;61:326-334.

4. Cockcroft JR. Exploring vascualr benefits of endothelium-derived nitric oxide. Am J Hypertension. 2005;18:1775-1835.

5. Nishimoto Y, Tomida T, Matsui H, Ito T, Okumura K. Decrease in renal medullary endothelial nitric oxide synthase of fructose-fed, saltsensitive hypertensive rats. Hypertension. 2002;40:190-194.

6. Huang PL, Huang Z, Mashimo H, et al. Hypertension in mice lacking the gene for endothelial nitric oxide synthase. Nature. 1995;377: 239-242.

7. Baylis C, Mitruka B, Deng A. Chronic blockade of nitric oxide synthesis in the rat produces systemic hypertension and glomerular damage. J Clin Invest. 1992;90:278-281.

8. Harrison D. Cellular and molecular mechanisms of endothelial cell dysfunction. J Clin Inv. 1997;100:2153-2157.

9. Panza JA, Quyyumi AA, Brush JE Jr, Epstein SE. Abnormal endotheliumdependent vascular relaxation in patients with essential hypertension. N Engl J Med. 1990;323:22-27.

10. Fisslthaler B, Dimmeler S, Hermann C, Busse R, Fleming I. Phosphorylation and activation of the endothelial nitric oxide synthase by fluid shear stress. Acta Physiol Scand. 2000;168:81-88.

11. Herrera M, Garvin JL. Recent advances in the regulation of nitric oxide in the kidney. Hypertension. 2005;45:1062-1067.

12. Nakane H, Miller FJ Jr, Faraci FM, Toyoda K, Heistad DD. Gene transfer of endothelial nitric oxide synthase reduces angiotensin II-induced endothelial dysfunction. Hypertension. 2000;35:595-601.

13. Zhao CX, Xu X, Cui Y, et al. Increased endothelial nitric-oxide synthase expression reduces hypertension and hyperinsulinemia in fructosetreated rats. J Pharmacol Exp Ther. 2009;328:610-620.

14. Gava AL, Peotta VA, Cabral AM, Vasquez EC, Meyrelles SS. Overexpression of eNOS prevents the development of renovascular hypertension in mice. Can J Physiol Parmacol. 2008;86:458-464.

15. Walker PD, Cavallo T, Bonsib MS. Practice guideline for the renal biopsy. Mod Pathol. 2004;17:1555-1563.

16. Zhou XJ, Laszik Z, Silva FG. Algorithmic approach to the interpretation of the renal biopsy. In: Zhou XJ, Laszik Z, Nadasdy T, D'Agati VD, Silva F, editors. Diagnostic Renal Pathology. New York, NY: Cambridge University Press; 2009:55-78. 
17. Zhao S, Gu X, Groome LG, Wang Y. Decreased nephrin and GLEPP-1, but increased VEGF, Flt-1, and nitrotyrosine, expressions in kidney tissue sections from women with preeclampsia. Reprod Sci. 2009;16:970-979.

18. Braam B, Verhaar MC. Understanding eNOS for pharmacological modulation of endothelial function: a translational view. Curr Pharm Des. 2007;13:1727-1740.

19. Lincoln TM, Corwell TL. Intracellular cyclic GMP receptor proteins. FASEB J. 1993;7:328-338.

20. Wright CE, Rees DD, Moncada S. Protective and pathological roles of nitric oxide in endotoxin shock. Cardiovasc Res. 1992;26:48-57.

21. Heeringa P, Steenbergen E, von Goor H. A protective role for endothelial nitric oxide synthease in glomerulonephritis. Kidney Int. 2002;61:822-825.

22. van Goor H, Albrecht EWJA, Heeringa $P$, et al. Nitric oxide inhibition enhances platelet aggregation in experimental anti-Thy-1 nephritis Nitric Oxide. 2001;5:525-533.

23. Gardiner SM, Compton AM, Bennett T, Palmer RM, Moncrda S Control of regional blood flow by endothelium-derived nitric oxide. Hypertension. 1990;15:486-492.

24. Jones LF, Brody MJ. Coronary blood flow in rats is dependent on the release of vascular nitric oxide. J Pharmacol Exp Ther. 1991;260:627-631.

25. Devlin AM, Brosnan J, Graham D, et al. Vascular smooth muscle cell polyploidy and cardiomyocyte hypertrophy due to chronic NOS inhibition in vivo. Am J Physiol. 1998;274:H52-H59.

26. Fisslthaler B, Dimmeler S, Hermann C, Busse R, Fleming I. Phosphorylation and activation of the endothelial nitric oxide synthase by fluid shear stress. Acta Physiol Scand. 2000;168:81-88.

27. Jarry A. Renaudin K, Denis MG, et al. Expression of NOS1 and soluble guanylyl cyclase by human kidney epithelial cells: Morphological evidence for an autocrine/paracrine action of nitric oxide. Kidney Int. 2003;64:170-180.

28. Furusu A, Miyazaki M, Abe K, et al. Expression of endothelial and inducible nitric oxide synthase in human glomerulonephritis. Kidney Int. 1998;53:1760-1768.

29. Kone BC, Kuncewicz T, Zhang W, Yu ZY. Protein interactions with nitric oxide synthases: controlling the right time, the right place, and the right amount of nitric oxide. Am J Physiol Renal Physiol. 2003;285: F178-F190.

30. Jiang JG, Chen RJ, Xiao B, et al. Regulation of endothelial nitric-oxide synthase activity through phosphorylation in response to epoxyeicosatrienoic acids. Prostaglandins Other Lipid Mediat. 2007;82:162-174.
31. Heltianu C, Costache G, Gafencu A, et al. Relationship of eNOS gene variants to diseases that have in common an endothelial cell dysfunction. J Cell Mol Med. 2005;9:135-142.

32. Marcun-Varda N, Zagradisnik B, Herodez SS, Kokalj VN, Gregoric A. Polymorphisms in four candidate genes in young patients with essential hypertension. Acta Paediatr. 2006;95:353-358.

33. Hsu YH, Hsu BG, Chen HI. Malignant alterations following early blockage of nitric oxide synthase in hypertensive rats. Chin J Physiol. 2007;50:283-293.

34. Heeringa P, Steenbergen E, van Goor H. A protective role for endothelial nitric oxide synthase in glomerulonephritis. Kidney Int. 2002;61: $822-825$.

35. Heeringa $\mathrm{P}$, van Goor $\mathrm{H}$, Moshage $\mathrm{H}$, et al. Expression of iNOS, eNOS, and peroxynitrite-modified proteins in experimental antimyeloperoxidase associated crescentic glomerulonephritis. Kidney Int. 1998;53:382-393.

36. Pfelischifter J, Schwarzenbach H. Interleukin 1 and tumor necrosis factor stimulate cGMP formation in rat renal mesangial cells. FEBS Lett. 1990;273:185-187.

37. Cattell V, Jansen A. Inducible nitric oxide synthase in inflammation. Histochem J. 1995;27:777-784.

38. Bollain-y-Goytia JJ, Ramirez-Sandoval R, Daza L, et al. Widespread expression of inducible NOS and citrulline in lupus nephritis tissue. Inflamm Res. 2009;58:61-66.

39. Kadkhodaee M, Zahmatkesh M, Sadeghipour HR, et al. Proteinuria is reduced by inhibition of inducible nitric oxide synthase in rat renal ischemia-reperfusion injury. Transplant Proc. 2009;41:2907-2909.

40. Chatterjee PK, Patel NS, Sivarajah A, et al. GW274150, a potent and highly selective inhibitor of iNOS, reduces experimental renal ischemia/ reperfusion injury. Kidney Int. 2003;63:835-865.

41. Li H, Forstermann U. Prevention of the atherosclerosis by interference with the vascular nitric oxide system. Curr Pharm Des. 2009;15: 3133-3145.

42. O'Connor DM, O'Brien T. Nitric oxide synthase gene therapy: progress and prospects. Expert Opin Biol Ther. 2009;9:867-878.

43. Gava AL, Peotta VA, Cabral AM, Vasquez EC, Meyrelles SS Overexpression of eNOS prevents the development of renovascualr hypertension in mice. Can J Physiol Pharmacol. 2008;86:458-464.

\section{Publish your work in this journal}

The International Journal of Nephrology and Renovascular Disease is an international, peer-reviewed open-access journal focusing on the pathophysiology of the kidney and vascular supply. Epidemiology, screening, diagnosis, and treatment interventions are covered as well as basic science, biochemical and immunological studies. The journal welcomes original

\section{Dovepress}

research, clinical studies, reviews \& evaluations, expert opinion and commentary, case reports and extended reports. The manuscript management system is completely online and includes a very quick and fair peerreview system, which is all easy to use. Visit http://www.dovepress.com/ testimonials.php to read real quotes from published authors. 